\title{
Single arc volumetric-modulated arc therapy is sufficient for nasopharyngeal carcinoma: a dosimetric comparison with dual arc VMAT and dynamic MLC and step-and-shoot intensity-modulated radiotherapy
}

Zhong-Hua Ning ${ }^{1}$, Jin-Ming Mu', Jian-Xue Jin ${ }^{2 *}$, Xiao-Dong Li ${ }^{3}$ Qi-Lin Li', Wen-Dong Gu', Jin Huang ${ }^{1}$, Yang $\mathrm{Han}^{1}$ and Hong-Lei Pei ${ }^{1,2^{*}}$

\begin{abstract}
Background: The performance of single arc VMAT (VMAT1) for nasopharyngeal carcinoma (NPC) on the Axesse linac has not been well described in previous studies. The purpose of this study is to assess the feasibility of VMAT1 for NPC by comparing the dosimetry, delivery efficiency, and accuracy with dual arc VMAT (VMAT2), dynamic MLC intensity-modulated radiotherapy (dIMRT), and step-and-shoot intensity-modulated radiotherapy (ssIMRT).

Methods: Twenty consecutive patients with non-metastatic NPC were selected to be planned with VMAT1, VMAT2, dIMRT and ssIMRT using Monaco 3.2 TPS on the Axesse ${ }^{\text {TM }}$ linear accelerator. Three planning target volumes (PTVs), contoured as high risk, moderate risk and low risk regions, were set to receive median absorbed-dose $\left(D_{50 \%}\right)$ of $72.6 \mathrm{~Gy}$, 63.6 Gy and 54 Gy, respectively. The Homogeneity Index (HI), Conformity Index (Cl), Dose Volume Histograms (DVHs), delivery efficiency and accuracy were all evaluated.

Results: Mean HI of PTV ${ }_{72.6}$ is better with VMAT1(0.07) and VMAT2(0.07) than dIMRT(0.09) and ssIMRT(0.09). Mean HI of PTV 63.6 is better with VMAT1 (0.21) and VMAT2(0.21) than dIMRT and ssIMRT. Mean Cl of PTV 72.6 is also better with VMAT1(0.57) and VMAT2(0.57) than dIMRT(0.49) and ssIMRT(0.5). Mean CI of PTV63.6 is better with VMAT1(0.76) and VMAT2(0.76) than dIMRT(0.73) and ssIMRT(0.73). VMAT had significantly improved homogeneity and conformity compared with IMRT. There was no significant difference between VMAT1 and VMAT2 in PTV coverage. Dose to normal tissues was acceptable for all four plan groups. VMAT1 and VMAT2 showed no significant difference in normal tissue sparring, whereas the mean dose of the parotid gland of dIMRT was significantly reduced compared to VMAT1 and VMAT2. The mean delivery time for VMAT1, VMAT2, dIMRT and ssIMRT was $2.7 \mathrm{~min}, 3.9 \mathrm{~min}, 5.7 \mathrm{~min}$ and $14.1 \mathrm{~min}$, respectively. VMAT1 reduced the average delivery time by $29.8 \%, 51.1 \%$ and $80.8 \%$ compared with VMAT2, dIMRT and ssIMRT, respectively. VMAT and IMRT could all be delivered accurately based on our quality assurance standards.

(Continued on next page)
\end{abstract}

\footnotetext{
*Correspondence: JianXue.Jin@elekta.com; hongleipei@126.com

${ }^{2}$ Department of Radiation Physics, Elekta China Co. Ltd, 27 North Fourth Ring

Mid Road, Chaoyang District, Beijing 100101, China

'Department of Radiation Oncology, The Third Affiliated Hospital, Soochow

University, 185 Juqian Road, Changzhou 213003, China

Full list of author information is available at the end of the article
} 
(Continued from previous page)

Conclusions: In the treatment of NPC using the Axesse ${ }^{\mathrm{TM}}$ linear accelerator, single arc VMAT has shown superiority to double arc VMAT, dIMRT and ssIMRT in delivery efficiency, without compromise to the PTV coverage. However, there is still room for improvement in terms of OAR sparing.

Keyword: Radiotherapy, Volumetric modulated arc therapy, Radiotherapy, Intensity modulated, Nasopharyngeal carcinoma, Plan evaluation, Dosimetry

\section{Background}

Nasopharyngeal carcinoma (NPC) is endemic, especially in southern China. Despite the rapid advancement in chemotherapy and molecular targeted therapy, radiotherapy is still the treatment of choice for NPC. Intensity-modulated radiation therapy (IMRT), the most commonly used in the treatment of NPC, has improved local control and 5-year survival rates with significantly lower radiation-induced toxicities than twodimensional radiotherapy $[1,2]$. However, the prolonged delivery time of IMRT poses two disadvantages. Firstly, the prolonged delivery time can decrease efficiency and increase intrafraction uncertainty of target volume localization and dosimetry $[3,4]$. Secondly, prolonging the fraction time will spare tissues with a fast DNA repair and might decrease tumor cell killing [5-7].

Volumetric-modulated arc therapy (VMAT) can generate precise conformal dose distribution through rotational delivery accompanied by variability of the Multileaf Collimator (MLC) position, dose rate and gantry rotation velocity $[8,9]$. Compared to IMRT, VMAT can improve the dose distribution, reduce the dose to normal tissues and shorten the delivery time [10]. It has been reported that dual arc VMAT for the treatment of NPC on a Synergy ${ }^{\text {тM }}$ (Elekta AB, Stockholm, Sweden) linear accelerator (linac) produced superior results in terms of Planning Target Volume (PTV) coverage and Organs at Risk (OARs) sparing, but was slightly less efficient than single arc VMAT [11]. Compared with Synergy ${ }^{\mathrm{TM}}$ linac, Axesse ${ }^{\mathrm{TM}}$ linac (Elekta AB, Stockholm, Sweden) is equipped with the improved Integrity ${ }^{\mathrm{TM}}$ treatment control system and the newly designed Agility ${ }^{\mathrm{TM}}$ head. This paper will address the question whether single arc VMAT, with aforementioned technical improvements, is adequate for NPC with no compromise PTV coverage and OARs sparing.

\section{Materials and methods}

\section{Patient selection and contouring}

This study was approved by ethical committee of Soochow University. 20 consecutive non-metastatic NPC patients were enrolled in this study between 2011 and 2012 at our department. For the purpose of comparability, the patient's VMAT and IMRT plans were all carried out by one experienced dosimetrist in the area of head and neck IMRT planning. The distribution of clinical stages according to the American Joint Committee on Cancer (AJCC) Staging System 2010 was as follows: Stage I, 1 (5\%); Stage II, 2 (10\%); Stage III, 11 (55\%); Stage IV, 6 (30\%).

All patients were immobilized with thermoplastic mask. Computed Tomography (CT) (Siemens Sensation ${ }^{\mathrm{Tm}}$, Munich, Germany) scan data (3 mm thickness, $512 \times 512$ pixels) was obtained from vertex to carina before imported into the Monaco 3.2 treatment planning system (TPS) (Elekta AB, Stockholm, Sweden).

In accordance with the Radiation Therapy Oncology Group (RTOG) 0225 and 0615 protocols [12,13], Gross Tumor Volume (GTV) covered gross tumor and regional metastatic nodes measuring more than $1 \mathrm{~cm}$ in diameter and/or showing central necrosis on the CT image. The $\mathrm{CTV}_{72.6}$ encompassed the GTV with a $0.5 \mathrm{~cm}$ margin; The $\mathrm{CTV}_{63.6}$, defined as the high-risk regions, included $\mathrm{CTV}_{72.6}$ and the entire nasopharynx, clivus, skull base, pterygoid fossae, parapharyngeal space, inferior sphenoid sinus, posterior third of the nasal cavity, maxillary sinuses as well as the retropharyngeal and upper deep jugular lymph node regions. Low jugular and supraclavicular regions with lymph node metastasis were also included in the $\mathrm{CTV}_{63.6}$. The $\mathrm{CTV}_{54}$, defined as the low-risk regions, included low jugular and supraclavicular regions without lymph node metastasis. The $\mathrm{CTV}_{72.6}, \mathrm{CTV}_{63.6}$ and $\mathrm{CTV}_{54}$ were all expanded by a $3 \mathrm{~mm}$ margin for $\mathrm{PTV}_{72.6}, \mathrm{PTV}_{63.6}$ and $\mathrm{PTV}_{54}$ to account for patient setup errors. The defined target volumes received three dose levels. The median absorbed-dose $\left(\mathrm{D}_{50 \%}\right)$ prescriptions were $72.6 \mathrm{~Gy} / 33 \mathrm{f}$ to $\mathrm{PTV}_{72.6}, 63.6 \mathrm{~Gy} /$ $33 \mathrm{f}$ to $\mathrm{PTV}_{63.6}$ and $54 \mathrm{~Gy} / 28 \mathrm{f}$ to $\mathrm{PTV}_{54}$. According to ICRU83 [14], the planning objectives included: $\mathrm{D}_{98 \%}$ (nearmin dose) of the $\mathrm{PTV}_{72.6}, \mathrm{PTV}_{63.6}$ and $\mathrm{PTV}_{54}$ are no less than $90 \%$ of planned absorbed dose, respectively. $\mathrm{D}_{95 \%}$ of the PTV $72.6, \mathrm{PTV}_{63.6}$ and $\mathrm{PTV}_{54}$ are no less than $95 \%$ of planned absorbed dose respectively. $\mathrm{D}_{2 \%}$ (near-max dose) of the PTV 72.6 is no more than $107 \%$ of planned absorbed dose respectively. $\left(D_{2 \%}, D_{98 \%}\right.$ and $D_{95 \%}$ represent the minimum absorbed dose received by $2 \%, 98 \%$ and $95 \%$ of the target volume, respectively). Regarding the OARs, the maximum doses to the brain stem and the spinal cord were set as 54 Gy and 45 Gy, respectively. In addition, at least one of the parotid glands should receive a mean dose of no more than $26 \mathrm{~Gy}$, or at least $50 \%$ of one gland should 
receive $<30$ Gy. The dose constraints to other normal tissues are all listed in Table 1.

\section{Planning technique}

VMAT and IMRT plans were all generated by Monaco 3.2 TPS on the Axesse ${ }^{\text {Tx }}$ linac. Coplanar beams using 6-MV photon were applied to all plans. On the Axesse ${ }^{\mathrm{Tax}}$ linac, Integrity $^{\text {ma }}$ supports Continuous Variable Dose Rate (CVDR) which makes the delivery of the prescription faster and smoother when compared to Banned Variable Dose Rate (BVDR) [15]. CVDR of the Axesse ${ }^{\text {Tx }}$ linac ranges from 45 $\mathrm{MU} / \mathrm{min}$ to $660 \mathrm{MU} / \mathrm{min}$. The thansmission and penumbra measurements of newly designed MLC of the Agility ${ }^{\text {max }}$ have a significant improvement compared to previous published data [16]. The Agility ${ }^{\text {mat }}$ has a160-leaf MLC of projected width $5 \mathrm{~mm}$ at the isocenter, designed to replace the tongue-and-groove system and allow for complete interdigitation and non-contiguous field shape. Instead of using a tongue-and-groove design to reduce interleaf leakage, the Agility ${ }^{\text {Tn }}$ slightly tilted the leaves relative to the actual beam divergence [17-19]. And due to the dynamic jaws orthogonal to the direction of leaf movement, leaf transmission is no more than $0.5 \%[16,20]$. Thanks to these designs, the Agility ${ }^{\text {tix }}$ has avoided tongue-and-groove effect, which may be easily seen on RapidArc [21]. The maximum speed of the dynamic leaf guide (DLG) is $3 \mathrm{~cm} / \mathrm{s}$. MLC maximum speed is $3.5 \mathrm{~cm} / \mathrm{s}$ and can approach $6.5 \mathrm{~cm} / \mathrm{s}$ with the aid of the DLG. The gantry maximum rotation velocity is $6 \%$. The minimum segment width was set at $5 \mathrm{~mm}$ with the minimum Monitor Units (MUs) of control points $(\mathrm{CPs})$ at $1 \mathrm{MU}$. The X-ray Voxel Monte Carlo (XVMC) algorithm is used for the final dose calculation and segment optimization [22], with a calculation grid of $3 \mathrm{~mm}$ and 3\% standard deviation.

\section{dIMRT and ssIMRT}

Both dIMRT and ssIMRT plans were generated with 9 equidistant coplanar beams uniformly distributed into $0^{\circ}$, $40^{\circ}, 80^{\circ}, 120^{\circ}, 160^{\circ}, 200^{\circ}, 240^{\circ}, 280^{\circ}, 320^{\circ}$. The maximum CPs of each angle was 15.

Table 1 Dose constraints for the critical structures

\begin{tabular}{|c|c|}
\hline OARs & Dose constraints \\
\hline Brain stem & Max dose <54 Gy \\
\hline Spinal cord & Max dose $<45$ Gy \\
\hline Parotid glands & Mean dose $<26$ Gy or $V_{30}<50 \%$ in one gland at least \\
\hline Chiasm & Max dose $<50$ Gy or $V_{54}<1 \%$ \\
\hline Optic nerves & Max dose $<50$ Gy or $V_{54}<1 \%$ \\
\hline Lens & Max dose $<10$ Gy \\
\hline Larynx & Mean dose < 45 Gy \\
\hline Oral cavity & Mean dose <45 Gy \\
\hline
\end{tabular}

Abbreviations: OARs; Organs at risk, Max dose; Maximum dose, $V x ; \%$ volume receiving $x \mathrm{~Gy}$.

\section{VMAT}

The rotating arc was set from $-180^{\circ}$ to $180^{\circ}$. The maximum CPs for VMAT1 was 180, and the maximum CPs per arc for VMAT2 was 120, which has been shown to be adequate for both efficiency and plan quality in our department.

\section{Dose-volume histograms (DVHs) and dose comparisons}

All the data is based on DVHs calculated using the Monaco 3.2 TPS (Elekta AB, Stockholm Sweden). The dosimetric comparison criteria were as follows:

1. Homogeneity Index (HI): used for evaluation of the PTV coverage by the prescription isodose. Formula: $\mathrm{HI}=\left(\mathrm{D}_{2 \%}-\mathrm{D}_{98 \%}\right) / \mathrm{D}_{50 \%}[23]$.

2. Conformity Index (CI): used for evaluation of the dose homogeneity within the PTV. Formula: $\mathrm{CI}=\left(\mathrm{TV}_{\mathrm{PV}} \times\right.$ $\left.\mathrm{TV}_{\mathrm{PV}}\right) /\left(\mathrm{V}_{\mathrm{PTV}} \times \mathrm{V}_{\mathrm{TV}}\right) .\left(\mathrm{V}_{\mathrm{TV}}\right.$ is the treatment volume of the body receiving $95 \%$ of the prescribed dose, $\mathrm{V}_{\mathrm{PTV}}$ is the volume of PTV, and $\mathrm{TV}_{\mathrm{PV}}$ is the volume of $V_{\text {PTV }}$ within the $V_{\text {TV }}$ ). CI value will be less than one, and the closer the CI to one, the better the conformality [24].

3. Delivery efficiency and dose verification: MUs and control points per fraction and plan calculation time for all plans were recorded. Treatment time was measured from beam-on to beam-off including time for radiation delivery and gantry rotation but not time for patient set-up. The mean dose rate was derived by dividing the MUs by beam-on time. Dosimetric validation was performed for all plans before being transferred to the Axesse ${ }^{\mathrm{Tm}}$ linac. The delivered dose was measured by a two-dimensional ionization chamber array MatriXX ${ }^{\mathrm{T}}$ (IBA Dosimetry, Schwarzenbruck, Germany). The calculated doses and the measured doses were compared by way of the Omnipro I'mRT software (IBA Dosimetry, Schwarzenbruck, Germany) which employs the gamma evaluation criteria of $3 \%$ and $3 \mathrm{~mm}$ [25].

4. Organs at risk: the normal tissue doses of both VMAT and IMRT plans were calculated. In particular, the $\mathrm{D}_{\max }$ (maximum point dose), $\mathrm{D}_{2 \%}$ (near maximum dose) and $\mathrm{D}_{\text {mean }}$ (mean dose) to serial organs were determined, as well as the $D_{\text {mean }}$ (mean dose) or $\mathrm{D}_{\mathrm{x} \%}$ (maximum dose encompassing $\mathrm{x} \%$ of the OARs volume) to parallel organs.

\section{Statistical analysis}

All analyses were performed using the SPSS version 17 statistical software (IBM SPSS Statistics). P-values are from two-sided tests. The normal distribution of variables was firstly checked with the Kolmogorov-Smirnov test. The differences in techniques were determined by the General Linear Model-univariate (GLM-u) procedure, 
and within-group differences between techniques were analyzed by post-hocmultiple comparisons LSD (Leastsignificant difference) method. $P<0.05$ was considered statistically significant.

\section{Results \\ PTV coverage}

Dose distribution in all VMAT and dIMRT plans for all 20 patients satisfied clinical requirements. Typical dose distributions of VMAT1, VMAT2, dIMRT and ssIMRT planned for one NPC patient are shown in Figure 1. The average DVHs of the PTVs is shown in Figure 2a. The $\mathrm{CI}$ and HI of the PTVs are shown in Table 2. It is found that both VMAT1 and VMAT2 showed superior dose homogeneity and conformity in PTVs compared to dIMRT and ssIMRT. However, there was no significant difference in target coverage between VMAT1 and VMAT2.

\section{Dose to the OARs}

The average dose and the maximum dose to the OARs for the $20 \mathrm{NPC}$ patients are listed in Table 3. The average DVHs to the OARs for the 20 NPC patients are shown in Figure $2 \mathrm{~b}-\mathrm{d}$. The four plan groups all meet well the requirements of the prescribed dose. The doses to normal tissues were within the clinically acceptable range. The four plan groups showed no significant difference in the doses to chiasm $D_{\max }$, left optic nerve $\mathrm{D}_{\text {max }}$ and right optic nerve $\mathrm{D}_{\text {mean }}$. In comparison with VMAT2, the $\mathrm{D}_{\text {mean }}$ of chiasm, left optic nerve and right lens in the VMAT1 plans was reduced, but the doses to spinal cord, oral cavity and larynx were increased. VMAT1 and VMAT2 showed no significant difference in normal tissue sparring. The dose to the right lens in the VMAT1 plans was reduced than that in the dIMRT and ssIMRT plans, while the doses to parotid gland and larynx in the VMAT1 plans were the opposite. The doses to oral cavity in the VMAT2 plans were lower than that in the dIMRT and ssIMRT plans, while VMAT2 plans had a higher dose to parotid gland compared to dIMRT plans.

\section{Delivery efficiency and dose verification}

The MUs, delivery time, mean dose rate, control points and plan calculation time of the four plan groups are all shown in Table 4. The MUs for the VMAT1, VMAT2, dIMRT and ssIMRT plans were 1232.1 \pm 146.2, 1349.9 $\pm 133.8,1292.2 \pm 120.7$ and 1090.2 \pm 91.1 , respectively. VMAT1 had lower MUs than VMAT2, and the MUs of ssIMRT Significantly reduced compared to both of VMAT. Delivery time for the VMAT1, VMAT2, dIMRT and ssIMRT plans were $2.7 \pm 0.2 \mathrm{~min}$, $3.9 \pm 0.3 \mathrm{~min}, 5.7 \pm 0.2 \mathrm{~min}$ and $14.1 \pm 1.0 \mathrm{~min}$, respectively. VMAT1 reduced the average delivery time by $29.8 \%$, $51.1 \%$ and $80.8 \%$ compared with VMAT2, dIMRT and ssIMRT, respectively. VMAT2 reduced the average delivery time by $30.2 \%$ and $72.3 \%$ compared with dIMRT and ssIMRT. The mean dose rates for the VMAT1, VMAT2, dIMRT and ssIMRT plans were $494.0 \pm 38.6 \mathrm{MU} / \mathrm{min}$, $379.6 \pm 30.1 \mathrm{MU} / \mathrm{min}, 249.8 \pm 17.9 \mathrm{MU} / \mathrm{min}$ and $86.6 \pm$ 5.1 MU/min, respectively. VMAT1 and VMAT2 increased the mean dose rate by $97.7 \%$ and $51.9 \%$ in comparison

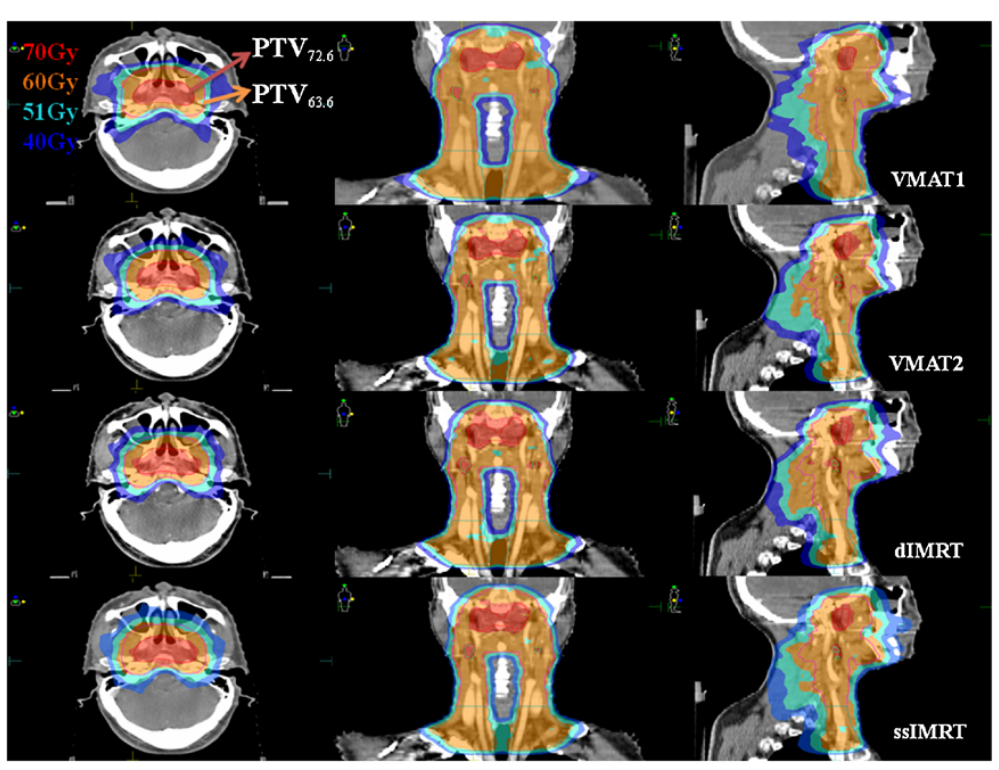

Figure 1 The dose distributions for one NPC patient planned for VMAT1 (top), VMAT2 (second), dIMRT (third) and ssIMRT (bottom). Color-wash areas: $70 \mathrm{~Gy}=$ red; $60 \mathrm{~Gy}=$ orange; $51 \mathrm{~Gy}=$ cyan; $40 \mathrm{~Gy}=$ blue; Red line is the outline of PTV 72.6 , wine and olive lines are the outlines of PTV $_{63.6}$. 
(a)

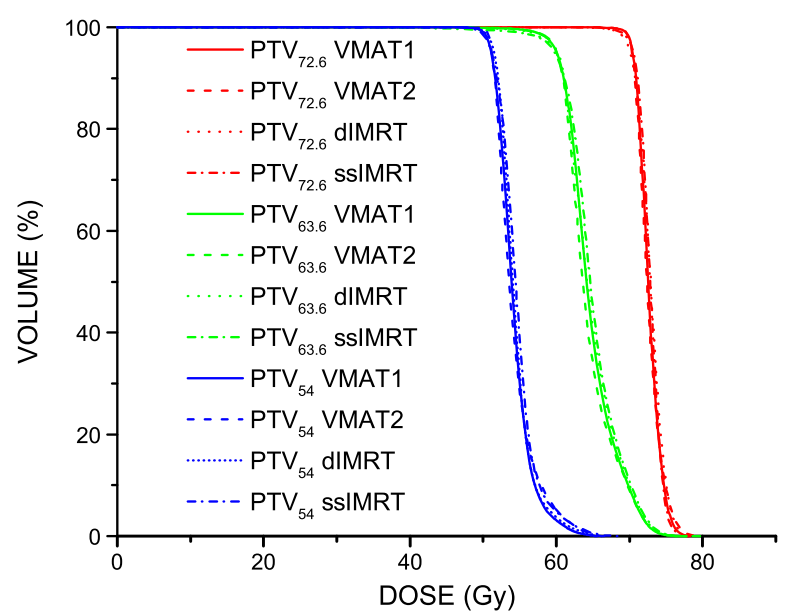

(c)

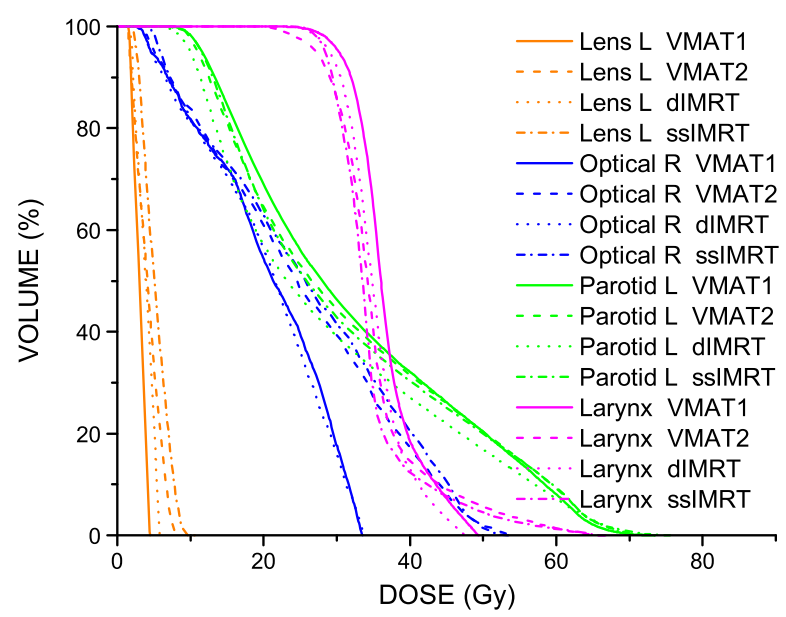

(b)

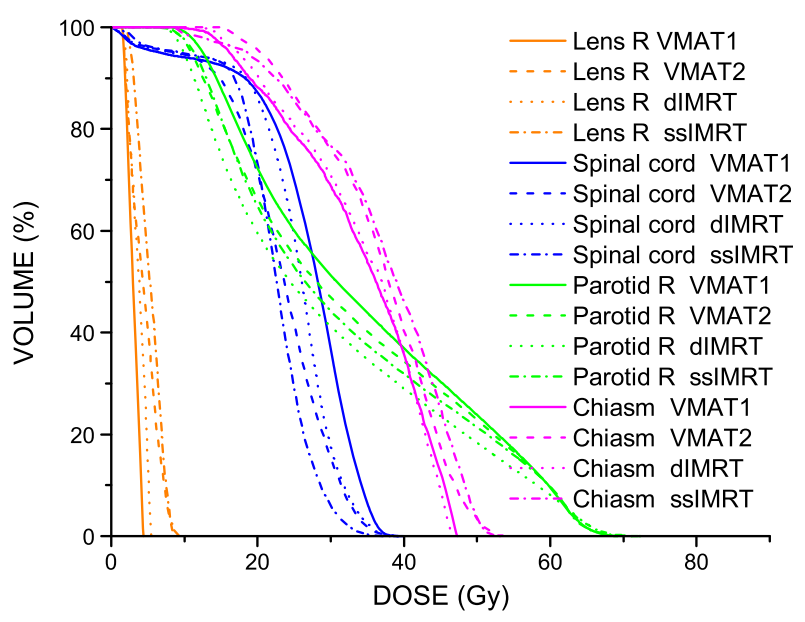

(d)

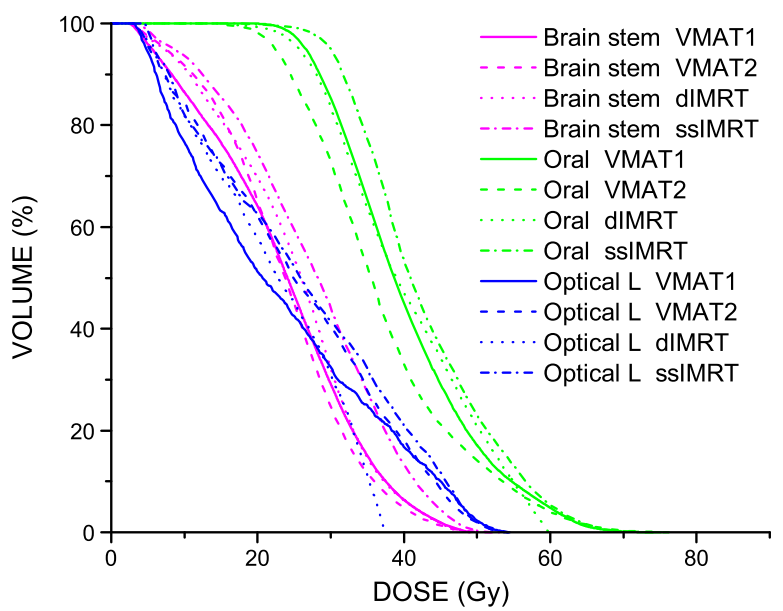

Figure 2 The average DVH to the OARs of 20 NPC patients. (a) The average DVH for all the PTVs comparing the four plan groups. (b-d) the average DVH to OARs of the four plan groups.

with dIMRT, and by $470.4 \%$ and $338.3 \%$ in comparison with ssIMRT. Dosimetric verification showed that all four techniques were accompanied by a high quality assurance. Average $\gamma$ pass rates for VMAT1, VMAT2, dIMRT and ssIMRT using the $3 \mathrm{~mm} / 3 \%$ gamma criteria were $96.3 \%$, $95.4 \%, 96.5 \%$ and $96.2 \%$, respectively.

\section{Discussion}

Target volume conformity and homogeneity are closely related to the complexity of target volumes, the delineation of volumes, the delivery equipment, the radiotherapy technology and the optimization algorithm. Single arc VMAT has been shown to successfully meet the clinical requirements of intensity modulation radiotherapy for simple target volume. For example, a recent study concluded that single-arc VMAT for prostate cancer was dosimetrically equivalent to fixed-beam IMRT
[26]. Most previous studies have indicated that, for complex target volumes such as those seen in head and neck cancer, single-arc VMAT may be less favorable dosimetrically than a fixed field IMRT $[11,27,28]$. This study has shown that target volume coverage and normal tissue sparing with VMAT1 is not significantly different from VMAT2. As expected, the target volume conformity and homogeneity of VMRT1 are better than that of IMRT.

In the past, it has been difficult to optimize single-arc VMAT for complex target volumes in head and neck cancer, especially NPC. When the optimization of a certain segment cannot meet the requirements, the TPS can rely on another arc to make up for the lack of intensity modulation. When single-arc VMAT was used in the treatment of NPC, smaller and faster MLCs, as well as the CVDR were sometimes needed to meet the plan optimization 
Table 2 Comparison of target volume homogeneity and conformity in all four radiotherapy plan groups

\begin{tabular}{|c|c|c|c|c|c|c|c|c|c|c|c|c|}
\hline & & VMAT1 & VMAT2 & dIMRT & ssIMRT & $p_{\mathrm{GLM}-\mathrm{u}}$ & $\begin{array}{l}p_{\text {VMAT1 }} \\
\text {-VMAT2 }\end{array}$ & $\begin{array}{l}p_{\text {VMAT1 }} \\
\text {-dIMRT }\end{array}$ & $\begin{array}{l}p_{\text {VMAT1 }} \\
\text {-ssIMRT }\end{array}$ & $\begin{array}{l}p_{\text {VMAT2 }} \\
\text {-dIMRT }\end{array}$ & $\begin{array}{l}p_{\text {VMAT2 }} \\
\text {-ssIMRT }\end{array}$ & $\begin{array}{l}p_{\text {dIMRT }} \\
\text {-ssIMRT }\end{array}$ \\
\hline \multirow[t]{6}{*}{$\mathrm{PTV}_{72.6}$} & $\mathrm{HI}$ & $0.07 \pm 0.01$ & $0.07 \pm 0.01$ & $0.09 \pm 0.01$ & $0.09 \pm 0.01$ & $<0.001$ & 0.510 & $<0.001$ & $<0.001$ & $<0.001$ & $<0.001$ & 0.653 \\
\hline & $\mathrm{Cl}$ & $0.57 \pm 0.09$ & $0.57 \pm 0.08$ & $0.49 \pm 0.07$ & $0.50 \pm 0.07$ & $<0.001$ & 0.955 & $<0.001$ & $<0.001$ & $<0.001$ & $<0.001$ & 0.355 \\
\hline & $\mathrm{D} 2 \%$ & $75.43 \pm 0.76$ & $75.40 \pm 0.89$ & $76.30 \pm 0.86$ & $76.57 \pm 0.66$ & 0.472 & & & & & & \\
\hline & D50\% & $72.64 \pm 0.03$ & $72.68 \pm 0.03$ & $72.93 \pm 0.11$ & $72.80 \pm 0.10$ & 0.588 & & & & & & \\
\hline & D95\% & $70.55 \pm 0.24$ & $70.69 \pm 0.30$ & $70.18 \pm 0.17$ & $70.10 \pm 0.23$ & 0.464 & & & & & & \\
\hline & D98\% & $70.01 \pm 0.45$ & $70.15 \pm 0.57$ & $70.01 \pm 0.44$ & $70.02 \pm 0.40$ & 0.742 & & & & & & \\
\hline \multirow[t]{6}{*}{ PTV 63.6} & $\mathrm{HI}$ & $0.21 \pm 0.02$ & $0.21 \pm 0.02$ & $0.23 \pm 0.02$ & $0.23 \pm 0.02$ & $<0.001$ & 0.427 & $<0.001$ & $<0.001$ & $<0.001$ & $<0.001$ & 0.614 \\
\hline & $\mathrm{Cl}$ & $0.76 \pm 0.03$ & $0.76 \pm 0.04$ & $0.73 \pm 0.03$ & $0.73 \pm 0.04$ & 0.001 & 0.448 & 0.003 & 0.001 & $<0.001$ & $<0.001$ & 0.605 \\
\hline & $\mathrm{D} 2 \%$ & $72.45 \pm 1.11$ & $72.42 \pm 1.26$ & $72.89 \pm 1.16$ & $73.20 \pm 0.79$ & 0.005 & 0.904 & 0.174 & 0.003 & 0.157 & 0.002 & 0.204 \\
\hline & D50\% & $64.14 \pm 0.28$ & $63.64 \pm 0.11$ & $63.99 \pm 0.19$ & $63.83 \pm 0.17$ & 0.236 & & & & & & \\
\hline & D95\% & $60.42 \pm 0.18$ & $60.44 \pm 0.12$ & $60.20 \pm 0.17$ & $60.17 \pm 0.27$ & 0.715 & & & & & & \\
\hline & D98\% & $58.49 \pm 0.32$ & $58.83 \pm 0.39$ & $57.99 \pm 0.50$ & $58.55 \pm 0.41$ & $<0.001$ & 0.007 & $<0.001$ & 0.651 & $<0.001$ & 0.021 & $<0.001$ \\
\hline
\end{tabular}

HI: homogeneity index; Cl: conformity index; GLM-u: general linear model-univariate; VMAT1: single arc VMAT, VMAT2: dual arc VMAT; dIMRT: dynamic MLC IMRT; ssIMRT: step-and-shoot IMRT. "Bold numbers" means that $p$ value has a statistically significance.

requirements. We noted that $10 \mathrm{~mm}$ width and $2 \mathrm{~cm} \mathrm{~s}^{-1}$ velocity MLCs without interdigitation and BVDR were mainly used in the previous studies $[11,27,28]$. Restricted by the mechanical components of linacs and MLCs, single-arc VMAT in these studies could not fully meet the intensity modulation requirements for the complex target volumes, therefore dosimetric distributions were inferior to double arc VMAT and IMRT. It has been reported that the $5 \mathrm{~mm}$ MLC width generates better treatment plans than the $10 \mathrm{~mm}$ MLC [29]. The Axesse ${ }^{\mathrm{rm}}$ linac can deliver CVDR and has much smaller and faster interdigitated MLCs, thus generating high quality plans by improving

Table 3 Dosimetric comparison of normal tissues in all four radiotherapy plan groups (Gy)

\begin{tabular}{|c|c|c|c|c|c|c|c|c|c|c|c|c|}
\hline & & VMAT1 & VMAT2 & dIMRT & ssIMRT & $p_{\mathrm{GLM}-\mathrm{u}}$ & $\begin{array}{l}p_{\text {VMAT1 }} \\
\text {-VMAT2 }\end{array}$ & $\begin{array}{l}p_{\text {VMAT1 }} \\
\text {-dIMRT }\end{array}$ & $\begin{array}{l}p_{\text {VMAT1 }} \\
\text {-ssIMRT }\end{array}$ & $\begin{array}{l}p_{\text {VMAT2 }} \\
\text {-dIMRT }\end{array}$ & $\begin{array}{l}p_{\text {VMAT2 }} \\
\text {-ssIMRT }\end{array}$ & $\begin{array}{l}p_{\text {dIMRT }} \\
\text {-ssIMRT }\end{array}$ \\
\hline \multirow[t]{2}{*}{ Brain stem } & $\mathrm{D} 2 \%$ & $41.21 \pm 0.92$ & $41.94 \pm 0.42$ & $42.56 \pm 0.48$ & $46.32 \pm 0.44$ & 0.024 & 0.679 & 0.444 & 0.005 & 0.724 & 0.015 & 0.036 \\
\hline & $\mathrm{D}_{\text {mean }}$ & $24.02 \pm 2.58$ & $23.46 \pm 3.97$ & $24.85 \pm 3.86$ & $27.51 \pm 0.41$ & $<0.001$ & 0.326 & 0.056 & $<0.001$ & 0.117 & $<0.001$ & $<0.001$ \\
\hline \multirow[t]{2}{*}{ Spinal-cord } & $\mathrm{D} 2 \%$ & $35.15 \pm 1.75$ & $32.59 \pm 2.91$ & $33.60 \pm 2.67$ & $28.66 \pm 3.02$ & $<0.001$ & 0.001 & 0.073 & $<0.001$ & 0.160 & $<0.001$ & $<0.001$ \\
\hline & $D_{\text {mean }}$ & $26.26 \pm 2.66$ & $23.46 \pm 2.74$ & $25.01 \pm 2.79$ & $22.3 \pm 3.52$ & $<0.001$ & $<0.001$ & 0.055 & $<0.001$ & 0.017 & 0.071 & 0.001 \\
\hline \multirow[t]{2}{*}{ Chiasm } & $\mathrm{D}_{\max }$ & $47.51 \pm 4.52$ & $47.89 \pm 5.01$ & $47.17 \pm 5.83$ & $48.23 \pm 6.32$ & 0.600 & & & & & & \\
\hline & $D_{\text {mean }}$ & $34.44 \pm 6.88$ & $36.38 \pm 5.79$ & $35.30 \pm 5.46$ & $36.37 \pm 7.30$ & 0.013 & 0.033 & 0.427 & 0.023 & 0.330 & 0.988 & 0.337 \\
\hline \multirow[t]{2}{*}{ Optic N L } & $D_{\max }$ & $43.73 \pm 10.41$ & $46.20 \pm 9.77$ & $44.13 \pm 9.52$ & $45.11 \pm 10.00$ & 0.177 & & & & & & \\
\hline & $\mathrm{D}_{\text {mean }}$ & $24.17 \pm 9.69$ & $26.17 \pm 9.69$ & $24.40 \pm 8.29$ & $26.22 \pm 9.24$ & 0.022 & 0.021 & 0.787 & 0.018 & 0.07 & 0.955 & 0.065 \\
\hline \multirow[t]{2}{*}{ Optic N R } & $D_{\max }$ & $43.30 \pm 11.85$ & $44.88 \pm 9.57$ & $42.04 \pm 10.18$ & $44.84 \pm 8.91$ & 0.048 & 0.177 & 0.278 & 0.188 & 0.017 & 0.971 & 0.018 \\
\hline & $D_{\text {mean }}$ & $25.04 \pm 9.69$ & $25.66 \pm 9.53$ & $23.92 \pm 8.74$ & $26.04 \pm 9.15$ & 0.076 & & & & & & \\
\hline Lens $L$ & $D_{\text {mean }}$ & $3.86 \pm 1.35$ & $4.13 \pm 1.49$ & $4.18 \pm 1.38$ & $5.18 \pm 1.53$ & $<0.001$ & 0.111 & 0.06 & $<0.001$ & 0.767 & $<0.001$ & $<0.001$ \\
\hline Lens $\mathrm{R}$ & $D_{\text {mean }}$ & $3.71 \pm 1.05$ & $4.35 \pm 1.7$ & $4.21 \pm 1.21$ & $5.22 \pm 1.38$ & $<0.001$ & 0.004 & 0.022 & $<0.001$ & 0.535 & $<0.001$ & $<0.001$ \\
\hline \multirow[t]{2}{*}{ Parotid L } & D50\% & $28.45 \pm 4.20$ & $27.04 \pm 4.99$ & $23.89 \pm 4.05$ & $26.73 \pm 4.09$ & $<0.001$ & 0.064 & $<0.001$ & 0.025 & $<0.001$ & 0.682 & $<0.001$ \\
\hline & $D_{\text {mean }}$ & $32.20 \pm 3.30$ & $31.57 \pm 3.86$ & $29.01 \pm 3.31$ & $31.48 \pm 3.21$ & $<0.001$ & 0.223 & $<0.001$ & 0.165 & $<0.001$ & 0.863 & $<0.001$ \\
\hline \multirow[t]{2}{*}{ Parotid R } & D50\% & $31.73 \pm 5.88$ & $28.18 \pm 5.38$ & $25.42 \pm 5.53$ & $27.07 \pm 3.79$ & $<0.001$ & 0.001 & $<0.001$ & $<0.001$ & 0.008 & 0.273 & 0.104 \\
\hline & $\mathrm{D}_{\text {mean }}$ & $34.14 \pm 3.56$ & $32.47 \pm 3.83$ & $30.11 \pm 3.93$ & $31.65 \pm 3.08$ & $<0.001$ & 0.085 & $<0.001$ & 0.001 & 0.001 & 0.238 & 0.097 \\
\hline Oral cavity & $D_{\text {mean }}$ & $40.35 \pm 4.77$ & $36.99 \pm 6.18$ & $41.42 \pm 6.77$ & $43.21 \pm 5.60$ & $<0.001$ & $<0.001$ & 0.230 & 0.002 & $<0.001$ & $<0.001$ & 0.049 \\
\hline Larynx & $\mathrm{D}_{\text {mean }}$ & $37.67 \pm 4.13$ & $35.17 \pm 4.55$ & $35.86 \pm 3.05$ & $34.76 \pm 3.82$ & $<0.001$ & 0.001 & 0.003 & $<0.001$ & 0.249 & 0.395 & 0.048 \\
\hline
\end{tabular}

GLM-u: general linear model-univariate; VMAT1: single arc VMAT, VMAT2: dual arc VMAT; dIMRT: dynamic MLC IMRT; ssIMRT: step-and-shoot IMRT. Lens L, left lens; Lens $\mathrm{R}$, right lens; Optic L, left optic nerve; Optic $\mathrm{R}$, right optic nerve; Parotid $\mathrm{L}$, left parotid; Parotid $\mathrm{R}$, right parotid. "Bold numbers" means that $p$ value has a statistically significance. 
Table 4 Comparison of delivery efficiency in all four radiotherapy plan groups

\begin{tabular}{|c|c|c|c|c|c|c|c|c|c|c|c|}
\hline & VMAT1 & VMAT2 & dIMRT & ssIMRT & $p_{\mathrm{GLM}-\mathrm{u}}$ & $\begin{array}{l}p_{\text {VMAT1 }} \\
\text {-VMAT2 }\end{array}$ & $\begin{array}{c}p_{\text {VMAT1 }} \\
\text {-dIMRT }\end{array}$ & $\begin{array}{l}p_{\text {VMAT1 }} \\
\text {-ssIMRT }\end{array}$ & $\begin{array}{c}p_{\text {VMAT2 }} \\
\text {-dIMRT }\end{array}$ & $\begin{array}{c}p_{\text {VMAT2 }} \\
\text {-ssIMRT }\end{array}$ & $\begin{array}{l}p_{\text {dIMRT }} \\
\text {-ssIMRT }\end{array}$ \\
\hline MUs & $1232.1 \pm 146.2$ & $1349.9 \pm 133.8$ & $1292.2 \pm 120.7$ & $1090.2 \pm 91.1$ & $<0.001$ & 0.001 & 0.067 & $<0.001$ & 0.079 & $<0.001$ & $<0.001$ \\
\hline Time (min) & $2.7 \pm 0.2$ & $3.9 \pm 0.3$ & $5.7 \pm 0.2$ & $14.1 \pm 1.0$ & $<0.001$ & $<0.001$ & $<0.001$ & $<0.001$ & $<0.001$ & $<0.001$ & $<0.001$ \\
\hline $\begin{array}{l}\text { Mean dose rate } \\
\text { (MU/min) }\end{array}$ & $494.0 \pm 38.6$ & $379.6 \pm 30.1$ & $249.8 \pm 17.9$ & $86.6 \pm 5.1$ & $<0.001$ & $<0.001$ & $<0.001$ & $<0.001$ & $<0.001$ & $<0.001$ & $<0.001$ \\
\hline Control points & $161.4 \pm 13.1$ & $187.0 \pm 30.9$ & $126.6 \pm 10.2$ & $138.0 \pm 10.4$ & $<0.001$ & $<0.001$ & $<0.001$ & $<0.001$ & $<0.001$ & $<0.001$ & 0.223 \\
\hline $\begin{array}{l}\text { plan calculation time } \\
\text { (hour) }\end{array}$ & $5.6 \pm 1.4$ & $4.8 \pm 1.2$ & $0.8 \pm 0.3$ & $0.8 \pm 0.4$ & $<0.001$ & 0.092 & $<0.001$ & $<0.001$ & $<0.001$ & $<0.001$ & 0.945 \\
\hline
\end{tabular}

GLM-u: general linear model-univariate; VMAT1: single arc VMAT, VMAT2: dual arc VMAT; dIMRT: dynamic MLC IMRT; ssIMRT: step-and-shoot IMRT. "Bold numbers" means that $p$ value has a statistically significance.

optimization significantly. Single-arc VMAT dosimetric distribution in this study was tailored to allow complex dose shaping around the NPC target volume. The results of this study has shown that single-arc VMAT, when applied to a complex target volume such as NPC using the Axesse $^{\mathrm{TM}}$ linac, is superior to IMRT and not inferior to VMAT2 for target coverage. And VMAT1 shortened delivery time and significantly improved delivery efficiency compared to VMAT2 and IMRT.

Previous results reported by $\mathrm{Lu}$ et al. [30] and White et al. [31] showed that double-arc VMAT for NPC achieved significant improvements in dose reduction to OARs and healthy tissue sparing compared with IMRT. On the other hand, Kan et al. [32] indicated that double-arc VMAT produced slightly inferior parotid sparing than nine-field "sliding-window" IMRT. A similar result was reported by Zhang et al. [28], who showed that the dose distribution of single-arc VMAT plan for NPC was slightly worse than that of a nine-field IMRT plan. In this study, we found that VMAT had improved plan quality in terms of target coverage compared to IMRT, but had no obvious advantage over IMRT in normal tissue sparing, even dIMRT showed an improved sparing of the parotid gland. The reported inconsistency might be related to the complexity of the target volume, the contouring of OARs and the dose constraints set. It is therefore difficult to draw a definite conclusion on single-arc VMAT for all NPC cases.

Similar to the previous VMAT studies [11,26,28], the delivery efficiency of VMAT in this study, especially VMAT1, was significantly superior to dIMRT and SSIMRT. VMAT1 reduced the average treatment time by $80.8 \%, 51.1 \%$ and $29.8 \%$ respectively compared with ssIMRT, dIMRT and VMAT2. When VMAT is applied in the treatment of NPC, delivery efficiency is related to the number of arcs, the dose rate and the MLC movement velocity. In vitro and in vivo experiments have shown that the prolonged delivery time shortened the tumor growth delay and survival time in tumor-bearing mice and the radiobiological effect decreased with the elongation of treatment time [33,34]. A shorter delivery time with VMAT1 could theoretically improve the radiobiological effect, however the clinical outcomes need further verification. As reported by Bertelsen et al. [35] and Cao et al. [15], the use of CVDR for VMAT reduced significantly the treatment time compared to BVDR. At the same time, it has been shown that beam-on time would decrease 10 to 40 seconds for every $100 \mathrm{MU} / \mathrm{min}$ increase of dose rate [36]. Therefore this study has shown that the optimized single-arc VMAT plan by using continuously variable high dose rate (mean dose rate $494 \mathrm{MU} / \mathrm{min}$ ), can improve delivery efficiency.

It has been reported that the MUs in VMAT plans for NPC cases were significantly lower than in IMRT plans $[11,32]$. In our study, VMAT plans improved delivery efficiency but did not significantly reduce MUs, compared with IMRT plans. To the authors' knowledge, the number of MUs for a given NPC plan is related to the adopted radiotherapy technique, TPS and the mechanical characteristics of the accelerator and MLCs.In comparison with other VMAT plans for NPC using the Monaco TPS [28], the number of VMAT MUs in this study was high for two reasons. Firstly, the optimized VMAT plan used a higher dose rate. The number of MUs increases with a higher dose rate, but the influence of a higher dose rate on the number of MUs decreases significantly with the increase of MLC velocity [37]. Similar reports have shown that a higher dose rate will increase the number of MUs and reduce the beam-on time in IMRT [36]. We can see in this study that the effect of a higher dose rate on the MUs didn't reduce the delivery efficiency of the VMAT plans. Secondly, the other reason for the increased MUs noted in VMAT plans is due to the interdigitation ability of the MLCs as well as threshold of the minimum segment width $(5 \mathrm{~mm})$ and the minimum CPs MU (1 MU). To be more specific, the optimized VMAT plans in this study generated a greater number of smaller and narrower segments, with less than 5 MUs in 20 percent of the CPs. Although this helped improve plan quality, it also increased the number of MUs. 


\section{Conclusions}

Based on an Axesse ${ }^{\mathrm{mm}}$ linear accelerator, single-arc VMAT for the treatment of NPC is entirely feasible, and furthermore provides better delivery efficiency than dualarc VMAT, dIMRT, and ssIMRT. Similar PTV coverage and sparing of OARs were observed in both VMAT delivery techniques. And both single-arc VMAT and dual-arc VMAT had improved target coverage compared to IMRT, but there is still room for improvement in terms of OAR sparing, such as parotid gland. The impact of reduced delivery time on clinical outcomes needs further investigation.

\section{Competing interests}

None of the authors report any competing interests.

\section{Authors' contributions}

HLP is responsible for the conception and study design. ZHN,WDG, JH, YH and HLP collected the data and contributed the cases. ZHN created the manuscript draft. JXJ, QLL and JMM supervised the medical physics. All authors read and approved the final manuscript.

\section{Acknowledgements}

The authors thank Jianxue Jin and his colleagues from Elekta China Co Ltd. for advance and guidance.

\section{Author details}

'Department of Radiation Oncology, The Third Affiliated Hospital, Soochow University, 185 Juqian Road, Changzhou 213003, China. '2Department of Radiation Physics, Elekta China Co. Ltd, 27 North Fourth Ring Mid Road, Chaoyang District, Beijing 100101, China. ${ }^{3}$ Department of Oncology, The Third Affiliated Hospital, Soochow University, 185 Juqian Road, Changzhou 213003, China.

Received: 27 May 2013 Accepted: 5 October 2013

Published: 14 October 2013

\section{References}

1. Lai SZ, Li WF, Chen L, Luo W, Chen YY, Liu LZ, Sun Y, Lin AH, Liu MZ, Ma J: How does intensity-modulated radiotherapy versus conventional twodimensional radiotherapy influence the treatment results in nasopharyngeal carcinoma patients? Int J Radiat Oncol Biol Phys 2011, 80:661-668.

2. Peng G, Wang T, Yang KY, Zhang S, Zhang T, Li Q, Han J, Wu G: A prospective, randomized study comparing outcomes and toxicities of intensity-modulated radiotherapy vs. Conventional two-dimensional radiotherapy for the treatment of nasopharyngeal carcinoma. Radiother Oncol 2012, 104:286-293.

3. Kim S, Akpati HC, Kielbasa JE, Li JG, Liu C, Amdur RJ, Palta JR: Evaluation of intrafraction patient movement for CNS and head \& neck IMRT. Med Phys 2004, 31:500-506.

4. Pan JJ, Zheng BH, Zhang Y, Chen CB, Li JL, Zhang X, Pan JJ, Zheng BH, Zhang $Y$, Chen CB, Li JL, Zhang XC: Measurement of setup error in conformal radiotherapy for nasopharyngeal carcinoma. Ai Zheng 2006, 25:115-118.

5. Mu X, Lofroth PO, Karlsson M, Zackrisson B: The effect of fraction time in intensity modulated radiotherapy: theoretical and experimental evaluation of an optimisation problem. Radiother Oncol 2003, 68:181-187.

6. Zheng XK, Chen LH, Wang WJ, Ye F, Liu JB, Li QS, Sun HW: Impact of prolonged fraction delivery times simulating IMRT on cultured nasopharyngeal carcinoma cell killing. Int J Radiat Oncol Biol Phys 2010, 78:1541-1547

7. Wang JZ, Li XA, D'Souza WD, Stewart RD: Impact of prolonged fraction delivery times on tumor control: a note of caution for intensitymodulated radiation therapy (IMRT). Int J Radiat Oncol Biol Phys 2003, 57:543-552.

8. Otto K: Volumetric modulated arc therapy: IMRT in a single gantry arc. Med Phys 2008, 35:310-317.
9. Yu CX, Tang G: Intensity-modulated arc therapy: principles, technologies and clinical implementation. Phys Med Biol 2011, 56:R31-R54.

10. Teoh M, Clark CH, Wood K, Whitaker S, Nisbet A: Volumetric modulated arc therapy: a review of current literature and clinical use in practice. Br J Radiol 2011, 84:967-996.

11. Lee TF, Ting HM, Chao PJ, Fang FM: Dual arc volumetric-modulated arc radiotherapy (VMAT) of nasopharyngeal carcinomas: a simultaneous integrated boost treatment plan comparison with intensity-modulated radiotherapies and single arc VMAT. Clin Oncol (R Coll Radiol) 2012, 24:196-207.

12. RTOG 0225 , a phase II study of intensity modulated radiation therapy (IMRT) +/chemotherapy for nasopharyngeal cancer. [http://www.rtog.org/ClinicalTrials/ ProtocolTable/StudyDetails.aspx?study=0225]

13. RTOG 0615, a phase I/ study of concurrent chemoradiotherapy using three dimentional conformal radiotherapy (3D-CRT) or intensity-modulated radiation therapy (IMRT) p bevacizumab (BV) for locally or regionally advanced nasopharyngeal cancer. [http://www.rtog.org/ClinicalTrials/ ProtocolTable/StudyDetails.aspx?study=0615]

14. International Commission on Radiation Units and Measurements: Planning Aims: Prescription, and technical data. J ICRU 2010, 10:55-59.

15. Cao D, Shepard D: The impact of continuous variable dose rate (CVDR) on VMAT plan quality, delivery efficiency and accuracy [abstract]. Med phys 2011, 38:3380-3381.

16. Cosgrove V, Thomas M, Weston S, Thompson M, Reynaert N, Evans C, Brown K, De Wagter C, Thwaites D, Warrington A: Physical characterization of a new concept design of an elekta radiation head with integrated 160-leaf multi-leaf collimator. Int J Radiat Oncol Biol Phys 2009, 75:S722-S723

17. Patel I, Glendinning AG, Kirby MC: Dosimetric characteristics of the elekta beam modulator. Phys Med Biol 2005, 50:5479-5492.

18. Bedford J, Thomas M, Smyth G, Warrington A: A pinnacle beam model for the agility 160-leaf multileaf collimator. Radiother Oncol 2012, 103:S322.

19. Bedford $J$, Thomas MD, Smyth G: Beam modeling and VMAT performance with the agility 160-leaf multileaf collimator. J App/ Clin Med Phys 2013, 14:172-185.

20. Schmidhalter D, Fix MK, Niederer P, Mini R, Manser P: Leaf transmission reduction using moving jaws for dynamic MLC IMRT. Med phys 2007, 34:3674-3687

21. Van Esch A, Huyskens DP, Behrens CF, Samsoe E, Sjolin M, Bjelkengren U, Sjostrom D, Clermont C, Hambach L, Sergent F: Implementing RapidArc into clinical routine: a comprehensive program from machine QA to TPS validation and patient QA. Med phys 2011, 38:5146-5166.

22. Fippel M: Fast monte carlo dose calculation for photon beams based on the VMC electron algorithm. Med phys 1999, 26:1466-1475.

23. International Commission on Radiation Units and Measurements: Special considerations regarding absorbed-dose and dose-volume prescribing and reporting in IMRT. J ICRU 2010, 10:27-40.

24. Feuvret L, Noel G, Mazeron JJ, Bey P: Conformity index: a review. Int J Radiat Oncol Biol Phys 2006, 64:333-342.

25. Bertelsen A, Hansen CR, Johansen J, Brink C: Single Arc volumetric modulated Arc therapy of head and neck cancer. Radiother Oncol 2010, 95:142-148.

26. Fontenot JD, King ML, Johnson SA, Wood CG, Price MJ, Lo KK: Single-arc volumetric-modulated arc therapy can provide dose distributions equivalent to fixed-beam intensity-modulated radiation therapy for prostatic irradiation with seminal vesicle and/or lymph node involvement. Br J Radiol 2012, 85:231-236.

27. Guckenberger M, Richter A, Krieger T, Wilbert J, Baier K, Flentje M: Is a single arc sufficient in volumetric-modulated arc therapy (VMAT) for complex-shaped target volumes? Radiother Oncol 2009, 93:259-265.

28. Zhang DD, Huang SM, Deng XW, Zhang GS: Comparison and evaluation of VMAT and IMRT for the treatment of initial treated nasopharyngeal carcinoma. Chinese J Radiat Oncol 2012, 21:364-368.

29. van Kesteren Z, Janssen TM, Damen E, van Vliet-Vroegindeweij C: The dosimetric impact of leaf interdigitation and leaf width on VMAT treatment planning in pinnacle: comparing pareto fronts. Phys Med Biol 2012, 57:2943-2952.

30. Lu SH, Cheng JC, Kuo SH, Lee JJ, Chen LH, Wu JK, Chen YH, Chen WY, Wen SY, Chong FC, et al: Volumetric modulated arc therapy for nasopharyngeal carcinoma: a dosimetric comparison with TomoTherapy and step-and-shoot IMRT. Radiother Oncol 2012, 104:324-330. 
31. White P, Chan KC, Cheng KW, Chan KY, Chau MC: Volumetric intensitymodulated arc therapy vs conventional intensity-modulated radiation therapy in nasopharyngeal carcinoma: a dosimetric study. J Radiat Res (Tokyo) 2013, 54:532-545.

32. Kan MW, Wong W, Leung LH, Yu PK, So RW, Cheng AC: A comprehensive dosimetric evaluation of using RapidArc volumetric-modulated arc therapy for the treatment of early-stage nasopharyngeal carcinoma. J Appl Clin Med Phys 2012, 13:189-202.

33. Wang $X$, Xiong XP, Lu J, Zhu GP, He SQ, Hu CS, Ying HM: The in vivo study on the radiobiologic effect of prolonged delivery time to tumor control in C57BL mice implanted with lewis lung cancer. Radiat Oncol 2011, 6:4.

34. Jiang L, Xiong XP, Hu CS, Ou ZL, Zhu GP, Ying HM: In vitro and in vivo studies on radiobiological effects of prolonged fraction delivery time in A549 cells. J Radiat Res (Tokyo) 2012, 54:230-234.

35. Bertelsen A, Lorenzen EL, Brink C: Validation of a new control system for elekta accelerators facilitating continuously variable dose rate. Med phys 2011, 38:4802-4810

36. Ghasroddashti E, Smith WL, Quirk S, Kirkby C: Clinical consequences of changing the sliding window IMRT dose rate. J App/ Clin Med Phys 2012, 13:3810

37. Vorwerk H, Wagner D, Hess CF: Impact of different leaf velocities and dose rates on the number of monitor units and the dose-volumehistograms using intensity modulated radiotherapy with sliding-window technique. Radiat Oncol 2008, 3:31.

doi:10.1186/1748-717X-8-237

Cite this article as: Ning et al: Single arc volumetric-modulated arc therapy is sufficient for nasopharyngeal carcinoma: a dosimetric comparison with dual arc VMAT and dynamic MLC and step-andshoot intensity-modulated radiotherapy. Radiation Oncology 2013 8:237.

\section{Submit your next manuscript to BioMed Central and take full advantage of:}

- Convenient online submission

- Thorough peer review

- No space constraints or color figure charges

- Immediate publication on acceptance

- Inclusion in PubMed, CAS, Scopus and Google Scholar

- Research which is freely available for redistribution 